\title{
Numerical Simulation on the Water-cooled Heat Sink for High-power Semiconductor
}

\author{
Liu Yanping \\ School of Energy, Power and Mechanical Engineering \\ North China Electric Power University \\ Beijing 102206
}

\begin{abstract}
With the development of electronic technology, the cooling of the electronic devices, especially for high-power semiconductor, becomes more and more important. The aircooled technology is gradually replaced by the water-cooled technology with the excellent cooling ability. This paper simulates the flow field and temperature field of water-cooled heat sink with FLUENT, obtains the heat transferring performance and hydrodynamics property of heat sink, and compares the simulation results with experimental results. The compared results find the disadvantages of heat sink structure and provide information for the improvement. The application of FLUENT, with the function of three-dimension modeling, hydro-dynamics calculation and heat transfer calculation, in the design of heat sink can improve the structure of heat sink, save manufacture cost, reduce exploitation time, and enhance the independent exploitation ability.
\end{abstract}

Keywords- FLUENT, Modeling, Numerical Simulation, Water-cooled Heat Sink

\section{INTRODUCTION}

In recent years, power electronic devices have begun to experience a significant expansion in various kinds of fields. With the development of electronic technology on manufacture technique and large-scale or super-large-scale integrated circuit, the method of cooling efficiency improvement becomes more and more important for the electronic equipments. The miniaturized and integrated electronic devices need transferring more heat on the unit area. The electronic device temperature will surpass the limited level and its performance will go down dramatically, if the heat cannot dissipate on time. Researches showed that with an ascension of $10^{\circ} \mathrm{C}$, the device reliability would drop to 50 percent [1]. Therefore, dissipating the surplus heat is the bottleneck of electronic device application. The cooling system must be taken into consideration in the design process of electronic equipments.

The objective of this paper is to simulate the working process of the water-cooled heat sink, which is often used in high-power electronic equipments. Although this kind of heat sink structure is complex, it is widely used in high power electronic equipments due to the higher heat transfer coefficient of water. However, there are many problems in the present design of water-cooled heat sink, due to the lack of design experience and out of line with the requirements of fluid mechanics and heat transfer. With the developments of

\author{
Yang Yuliang \\ School of Energy, Power and Mechanical Engineering \\ North China Electric Power University \\ Beijing 102206
}

CFD and computer technology, the simulation of flow and heat transfer process becomes reliable. Thus, this paper simulates the working process of water-cooled heat sink in order to optimize its design.

\section{THE COOLING PROGRAMME OF HIGH-POWER ELECTRONIC DEVICES}

In the thermal design of electronic devices, through the selection of heat dissipation method, a low cost and high efficient structure can be developed for the high-power electronic device. The structure is very important to ensure the internal junction temperature of electronic component in the allowable range [2].

According to the actual total power of IGBT (Insulated Gate Bipolar Transistor, about $3 \sim 6 \mathrm{kw}$ ), the traditional cooling methods are difficult to satisfy their basic requirements. So, our research adopts the liquid cooling method, i.e. Closed Loop Water-cooled System. Its principle is that the cooling water from the pump flows through the heat sink contacted with IGBT, so that the heat can be transferred between the cooling water and the IGBT. The water-cooled heat sink is the key factor that affects the cooling ability of the whole system directly. The main advantage of this design is its efficient transferring heat ability. Specific structural model of this design is shown in Figure 1.

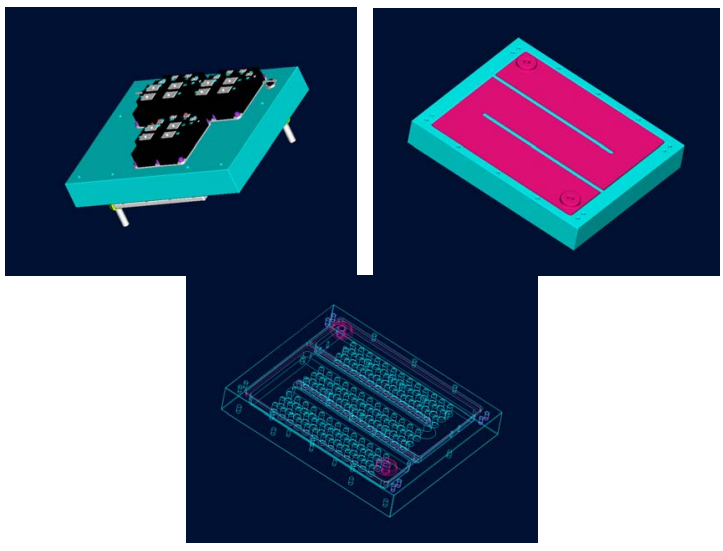

Figure 1. Model of Water-cooled Heat Sink 


\section{NUMERICAL SIMULATION}

\section{A. building of model}

The heat resource is three IGBT chips, whose power is totally $3.1 \mathrm{kw}$. The basic geometric structure of the heat sink is the cuboid with many spoiler columns in the chamber. The functions of the spoiler columns are to enhance turbulence flow and enlarge the heat transferring area between heat sink and cooling water [3]. Then, the spoiler column will reduce the heat resistance and improve heat dissipation efficiency. Heat sink is made of aluminum.

The basic external dimensions of the model are listed as following:

1) The length, width and height of the heat sink model are $420 \mathrm{~mm}, 340 \mathrm{~mm}$, and $60 \mathrm{~mm}$, respectively. In the front surface, there are inlet and outlet of water as shown in Figure 1.

2) Fluid model, or chamber model of heat sink, is a approximate cuboid. Its length, width and height are $340 \mathrm{~mm}$, $290 \mathrm{~mm}$, and $25 \mathrm{~mm}$, respectively. Its external surface is completely coupled with the internal surface of heat sink and its structure is shown in Figure 2 and 3.

3) The length, width and height of IGBT heat source model are $140 \mathrm{~mm}, 130 \mathrm{~mm}$, and $38 \mathrm{~mm}$, respectively. There are three IGBT models in the center of the heat sink back with the same size as shown in Figure 1.

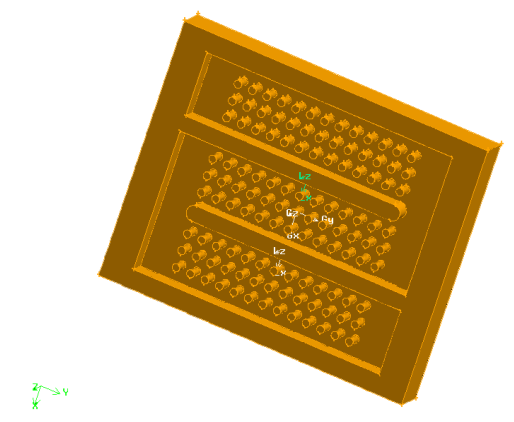

Figure 2. The Picture of Inside Structure

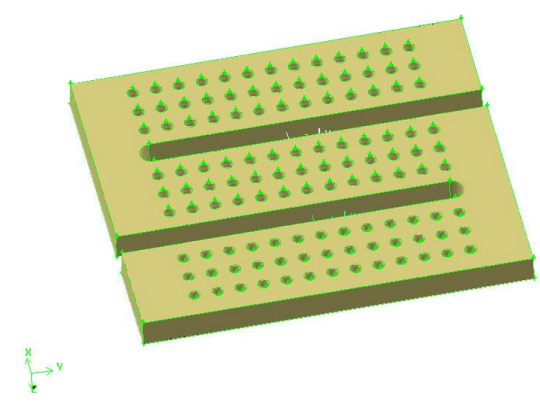

Figure 3. The Picture of Fluid Model

This research uses the GAMBIT to build the model which is divided into three parts: fluid model, heat sink model, and IGBT model. However, the function of GAMBIT is not plentiful enough to build complex original model. So, only a simplified model is built here.

\section{B. meshing}

In this research, the tetrahedral solid elements and fivesided (and pyramid-shaped) body elements are adopted. The meshing method is Tgrid, which can control the density of elements and select the tetrahedral solid elements or fivesided (and pyramid-shaped) body elements according to geometric shape of model automatically. Taking into account of the computaion ability of our computer, the model is finally divided into 450,000 grids [4].

\section{C. setting boundary conditions}

There are two steps to set boundary conditions in FLUENT. Firstly, we set fluid model material as liquid and heat sink material as solid in pre-processing softwareGAMBIT[5]. Secondly, we set other boundary conditions such as inlet, outlet and wall in the fluid area. After importing the model into the FUIENT, specific parameters can be set as follows.

1) The viscous model should choose as " $k-\omega$ " and keep its default setting.

2) The energy equation should be available. The energy equation will be used in the coupled computation of fluid mechanic and theory of heat transfer, which are two aspects involved in the computation.

3) IGBT heat resource material should be aluminum, which is the body heat resource. Its heating power density can be written as:

$$
q=\frac{3100}{0.13 \times 0.14 \times 0.038}=4482 \mathrm{Kw} / \mathrm{m}^{3}
$$

4) The heat sink model material should be aluminum.

5 ) The fluid model material should be "water-liquid".

6 ) The inlet should be set as "velocity inlet". The velocity of water in inlet is set as $1.8863 \mathrm{~m} / \mathrm{s}$, temperature is set as $298 \mathrm{k}$, turbulent intensity is set as $10 \%$, and hydraulic diameter is set as $12.5 \mathrm{~mm}$.

7) The outlet should be set as "outflow". The outflow condition should be set in the bound of outlet to limit the fluid pouring only within this range.

8) The external surface of heat sink and IGBT should be "wall", whose heat property should be set as "convection", in order to exchange heat with surrounding air. The heat transfer coefficient is usually $5-25 \mathrm{w} /(\mathrm{m} 2 \mathrm{k})$. We set the value of this parameter as $20 \mathrm{w} /(\mathrm{m} 2 \mathrm{k})$.

\section{CALCULATION and analysis of the simulation results}

The fluid mechanics model is three-dimension steady incompressible viscous flow. Many fluid control equations are involved in the numerical computation process, such as the continuity equation, momentum equation, the turbulent kinetic energy equation, the turbulent diffusion equation, and the energy equation. After the computation, the residual of each variable has been reduced to 0.001 , in accordance with the criteria. The results are shown fromFigures 4 to 9 . 

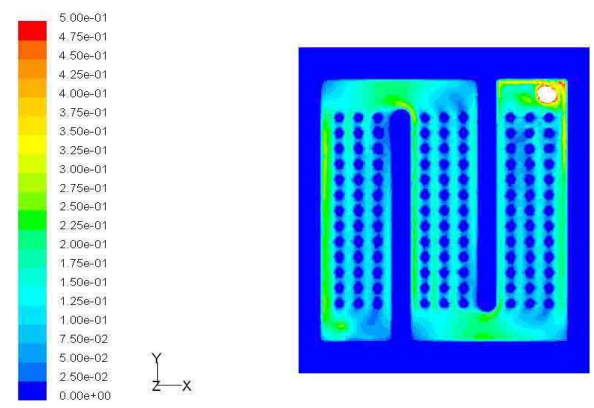

Figure 4. The Distributing Picture of Velocity of Flow Field (from $0 \mathrm{~m} / \mathrm{s}$ to $0.5 \mathrm{~m} / \mathrm{s}$ )

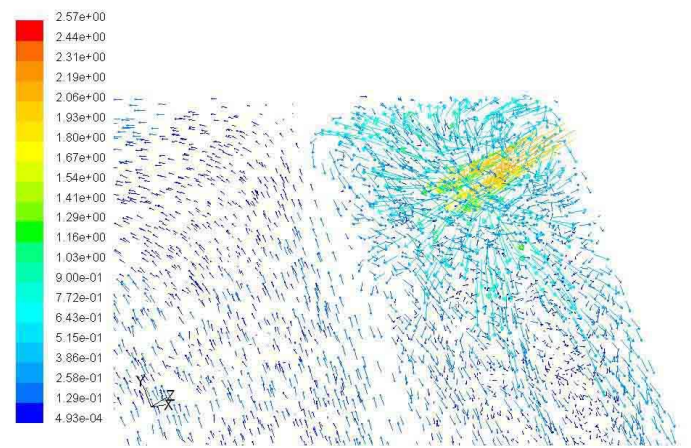

Figure 5. The Distributing Picture of Velocity Vectors of Inlet Field
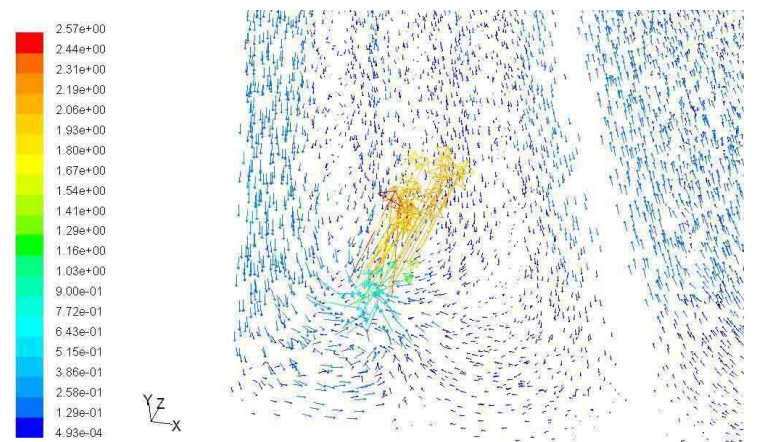

Figure 6. The Distributing Picture of Velocity Vectors of Outlet Field $(\mathrm{m} / \mathrm{s})$
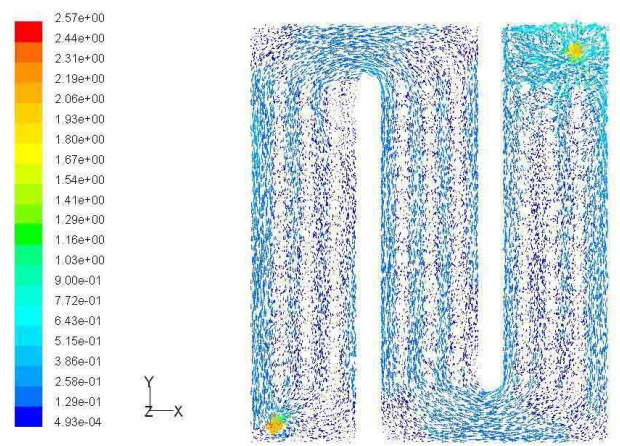

Figure 7. The Distributing Picture of Velocity Vectors of Flow Field
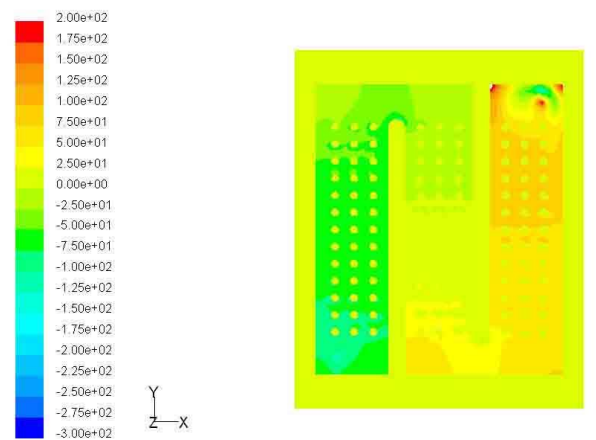

Figure 8. The Distributing Picture of Static Pressure of Flow Field (Pa)
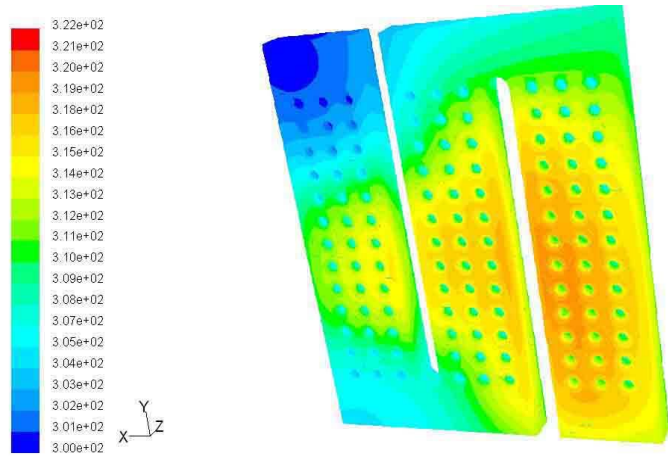

Figure 9. The Distributing Picture of Temperature of Flow Field

According to Figure 9, the fluid temperature difference between inlet and outlet is about 3 degrees. The temperature of the area near IGBT is about $51{ }^{\circ} \mathrm{C}$. The experimental value is $53.6{ }^{\circ} \mathrm{C}$. So, the simulative value is convinced. After analyzing the simulation results through post-processing tools of FLUENT, the data shows that fluid convection brings out heat at about $3007 \mathrm{w}$, while $93 \mathrm{w}$ heat is transferred by air convection. So, more than $96 \%$ heat is transferred by water flow. Thus, the design of heat sink is successful. However, there are still some problems listed as follows.

According to the plot of fluid velocity distribution (Figure 4) and pressure distribution (Figure 8), the velocity of flow around the vertical gap of spoiler column is high, while the velocity of flow around the horizontal gap of spoiler column and each dead angle is low.

The plot of vector distribution of the fluid flow (Figure 7) shows that there is no vortex. Many spoiler columns are set in the chamber of heat sink, which is not the same as the actual turbulence. The spoilers cause a high vertical gap velocity and low horizontal velocity.

The heat transfer intensity of fluid convection is mainly affected by factors of the type of fluid, velocity, flow status and transferring heat area. The goal of this design is to find a 
balance point among the factors involved above, so as to achieve the best cooling effect.

According to the problems listed above, the structure of heat sink can be improved through following aspects:

If we change the order arrangement of spoiler column into cross arrangement, the intensity of turbulence will be enhanced, and the effect of heat transfer improves naturally.

The reduction of the number of spoiler columns leads to the higher average velocity of internal water, reduces the area of heat transfer, and affects the heat transfer effect.

The adjustment of the dead angle with 45 degree will eliminate the phenomenon of the low velocity and detention of water in the dead angle.

\section{CONCLUSIONS}

This paper simulated the water-cooled heat sink threedimension flow field and temperature field by FLUENT and achieved the unique three-dimension graphic display of flow velocity, pressure, temperature and other parameters. The simulation results showed the transferring heat ability of heat sink and characteristics of flow mechanics, which can be used to guide the optimization design of heat sink internal structure. The proposed method can also help to change the situation that engineers relied on experiments to obtain data for such design.

\section{REFERENCES}

[1] J.P.Holman . Heat Transfer. China Machine Press[M], 2005

[2] T.Y. Lee. Design Optimisation of An Integrated Liquid-cooled IGBT Power Module Using CFD Technique. Proc. Sixth Annual Conference on Thermal and Thermo-mechanical Phenomena in Electronic Systems[C], 1998,5,PP:337-342

[3] LIU Yan-ping; GAO Xin-xia. Numerical Simulation on the Heat Sink for High-power semiconductor. Chinese journal of electronic device [J], 2007,2, PP: 608-611

[4] C.S. Yun; P. Malberti. Thermal Component Model for Electrothermal Analysis of IGBT Module Systems. IEEE Trans. on Advanced Packaging[J], 2008, 3,pp:401-406.

[5] WANG Fu-jun.Analysis of Computational Fluid Dynamics. Tsinghua University Press[M], 2004.9

[6] ZHANG Xi-min; REN Ze-xu; MIN Fei-ming.Heat Transfer (the forth edition).China Building Industry Press[M], 2001

[7] LI Wan-ping.Computational Fluid Dynamics. Huazhong University of Science and Technology Press [M], 2005 